\title{
UNCERTAINTY ANALYSIS OF A WEB-BASED DATA ACQUISITION SYSTEM FOR POULTRY MANAGEMENT WITH SENSOR NETWORKS
}

\author{
Ligen Yu ${ }^{1,4}$, Guanghui Teng ${ }^{2}$, Gerald L. Riskowski ${ }^{3}$, Xuzhang $\mathrm{Xu}^{1}$, Wenzhong Guo ${ }^{*}$
}

${ }^{5 *}$ Corresponding author. National Research Center of Intelligent Equipment for Agriculture, Key Laboratory of Agricultural Intelligent Equipment Technology, Beijing, China. E-mail: guowz@nercita.org.cn

\section{KEYWORDS}

Uncertainty analysis, Sensor networks, Data acquisition, Measurement, Poultry management.

\begin{abstract}
This paper presents a web-based data acquisition system developed by the LabVIEW software program for environment monitoring for poultry management. The measurement error and uncertainty analysis should be conducted accurately to maximize the reliability of this system. An algorithm was proposed for the uncertainty analysis to estimate the sensor networks, with the calibration and validation processes fulfilling the standards of the Guide to the Expression of Uncertainty in Measurement. For the different environmental sensors, the values of the uncertainties were calculated by the methods of type A and B evaluation through a case study. Environmental parameters including air temperature, relative humidity, and $\mathrm{CO}_{2}$ concentration of a compartment housing for a small group $(\mathrm{n}=90)$ of laying hens were measured using a perch aviary system in a 24-h period with an interval of $5 \mathrm{~min}$. The results showed that with the perch system for laying hens, the expanded uncertainty of the data acquisition system was above $1.02{ }^{\circ} \mathrm{C}, 5.54 \%$, and $67.8 \mathrm{ppm}$ over the sensor data of the air temperature, relative humidity, and $\mathrm{CO}_{2}$ concentration, respectively. Moreover, the relative uncertainty of this system was estimated as $15.9 \%$. Therefore, because of its more reliable uncertainty analysis and data evaluation, the proposed web-based data acquisition system has considerable potential for ensuring correct decision-making when used in poultry production.
\end{abstract}

\section{INTRODUCTION}

In recent years, the improvement of informative networks and sensor technologies has enabled the rapid growth of wireless and online management systems. These systems with sensor networks can remotely monitor and maintain communication with many unfavorable physical environments such as remote geographic regions, inaccessible dangerous locations, and commercial poultry farms (Georgiadis et al., 2009; So-In et al., 2014; Venkatraman et al., 2016; Zahedi et al., 2016). The management systems based on networks have many wide applications, ranging from earth observations (Datla et al., 2010) to agricultural production (Serodio et al., 2001; Brunner et al., 2012). Numerous sensor networks are used to measure the environment and directly guide production procedures. Thus, it is important to evaluate the measurement uncertainty and sensor network errors with a web-based data acquisition and remote management system so that the uncertainty of the system can be estimated correctly (Kessel et al., 2008; Pechlivanidis et al., 2011; Sarachi et al., 2015). Moreover, analyzing the sensor data will increase the confidence of the authorities in the results to make decisions.

The requirements for error evaluation led to the publication in 1993 of the first editions of the standards of the "Guide to the Expression of Uncertainty in Measurement (GUM)" by the International Organization for Standardization (ISO) in collaboration with the International Bureau of Weights and Measures (BIPM), International Electrotechnical Commission (IEC), International Laboratory Accreditation Conference (ILAC), and many other organizations. This formally established the general rules for evaluating and expressing the uncertainties in a broad spectrum of measurements (ISO, 1995; BIPM, 2015; Ellison \& Williams, 2012). With reference to increased need for accurate measurements, a Joint Committee for Guides in Metrology

\footnotetext{
${ }^{1}$ National Research Center of Intelligent Equipment for Agriculture, Key Laboratory of Agricultural Intelligent Equipment Technology, Beijing, China.

${ }^{2}$ Key Laboratory of Agricultural Engineering in Structure and Environment, Ministry of Agriculture, China Agricultural University, Beijing, China.

${ }^{3}$ Texas A\&M University/College Station, Texas, USA.

${ }^{4}$ Beijing Engineering Research Center for Information Technology in Agriculture, P.R. China.

Received in: 9-28-2017
}

Accepted in: 10-15-2018 
(JCGM, 2015) was appointed to promote the use of GUM. Moreover, it had to give added referenced documents to GUM through guidance on the aspects of the evaluation and use of the measurement uncertainty, which are not explicitly treated in the 1997 GUM. For the application of this guide, a series of supplementary documents including JCGM 100 (2008), JCGM 101 (2008), JCGM 102 (2011), and JCGM 200 (2012) were released by the JCGM. Moreover, in China, a technical specification named "Evaluation and Expression of Uncertainty in Measurement (JJF 1059-1999)" was issued by the General Administration of Quality Supervision (GAQS, 1999), Inspection, and Quarantine of China in 1999, and a new revised guide for the measurement uncertainty (JJF1059.1-2012) was released and used for various industries (Huang et al., 2011; Chen et al., 2016). These guides and specifications provide detailed guidance for the evaluation and expression of the uncertainty in quantitative analysis based on the approach adopted in ISO (GUM) and the AQSIQ (JJF).

In principle, numerous model simulations and calculation procedures have been reported for the uncertainty evaluation in measurements. These include the Monte Carlo method (Yustres et al., 2012), Fuzzy sets theory, Grey system theory, and Bayesian theory (Reilly \& Willenbockel, 2010; Broekhuizen et al., 2015). These evaluation models are primarily developed with the aim of successfully finding a single best set of parameter values. The focus is on the steps of deriving a corresponding measurement model, the assignment of the probability distribution functions (PDFs) to the input parameters in the model, and the propagation of the distributions through the model (Zakharov et al., 2013; Snow \& Bajaj, 2015). Therefore, in numerous studies, the procedure for calculating the arithmetic mean of the initial quantities followed by conversion into an estimate of the measured quantity yields a shifted estimate because of their nonlinear relationship. From the comparison of these models, the methods of type $\mathrm{A}$ and $\mathrm{B}$ evaluation following the standards of GUM and JJF seem to be best suited for conducting the data evaluation and consistency checking of a data acquisition system with sensor networks. A sample study illustrated how the method can be implemented.

This article aimed to demonstrate a web-based data acquisition system and the entire process of the uncertainty analysis for sensor networks. The specific objectives of this work were: (1) to introduce a web-based data acquisition system using the LabVIEW software program to measure environmental parameters for poultry management, (2) to deliver an algorithm for the uncertainty analysis of various sensor data based on the methods of type A and B evaluation, and (3) to examine the system performances based on the uncertainty analysis of the sensor network.

\section{SYSTEMS DESCRIPTION}

\section{Overview of the Hardware of the Data Acquisition System}

The hardware of the data acquisition system (short for System 1) mainly consisted of various sensors, signal conversion modules, and a datalogger embedded, and its software was a data acquisition program, as presented in Fig. 1. The data acquisition module was designed to collect the environmental parameters including air temperature, relative humidity, and $\mathrm{CO}_{2}$ concentration of a compartment housing for laying hens. The power supply and digital output of the sensors were $24 \mathrm{~V}$ DC and 4-20 mA. Other sensors with the same signal output can also be feasible for this system. The features of the sensors used in this system are listed in Table 1.

The key component of this system is the signal conversion module (Model DAM-E3058F, Art Technology Development Ltd, Beijing, China) with the following specifications: 8 channels of analog output, $10 \mathrm{~Hz}$ sampling rate, and 16-bit resolution. The module interacts with the data server through web request based on the Modbus Communication Protocol. It allows the system to read out the values from the sensors and transfer the signal values to measured values using a linear interpolation method.

The key component of this system is the signal conversion module (Model DAM-E3058F, Art Technology Development Ltd, Beijing, China) with the following specifications: 8 channels of analog output, $10 \mathrm{~Hz}$ sampling rate, and 16-bit resolution. The module interacts with the data server through web request based on the Modbus Communication Protocol. It allows the system to read out the values from the sensors and transfer the signal values to measured values using a linear interpolation method.

TABLE 1. Features of the sensors used in this system.

\begin{tabular}{|c|c|c|c|c|c|}
\hline & $\begin{array}{c}\text { Model } \\
\text { Number }\end{array}$ & Manufacturer & $\begin{array}{c}\text { Measurement } \\
\text { Range }\end{array}$ & Accuracy & $\begin{array}{c}\text { Long-term } \\
\text { Stability }\end{array}$ \\
\hline $\begin{array}{l}\text { Air temperature } \\
\text { sensor }\end{array}$ & JWICV & ColliHigh Sensor Technology, Co., Ltd, & $-40-120^{\circ} \mathrm{C}$ & $\pm 0.5^{\circ} \mathrm{C}$ & $0.1^{\circ} \mathrm{C} / \mathrm{yr}$ \\
\hline $\begin{array}{l}\text { Relative humidity } \\
\text { sensor }\end{array}$ & JWSK-6 & Beijing, China & $0-100 \%$ & $\pm 2 \%$ & $<1 \% / \mathrm{yr}$ \\
\hline $\begin{array}{l}\mathrm{CO}_{2} \text { concentration } \\
\text { sensor }\end{array}$ & VC1008T & Telasia Symtonic Pte Ltd, Singapore & 0-2000 ppm & $\begin{array}{l} \pm 30 \text { ppm } \pm 2 \% \\
\text { reading }\end{array}$ & $<2 \% / 10$ yrs \\
\hline
\end{tabular}

The Ethernet router (HT-3GW, HLWT-TECH Ltd, Beijing, China) is used to establish communications with remote sites based on Third-Generation Wireless Networks $(3 \mathrm{G})$, as shown in Fig. 1. The terminal users connect to the system for monitoring the environmental condition and determine the poultry performance remotely. 


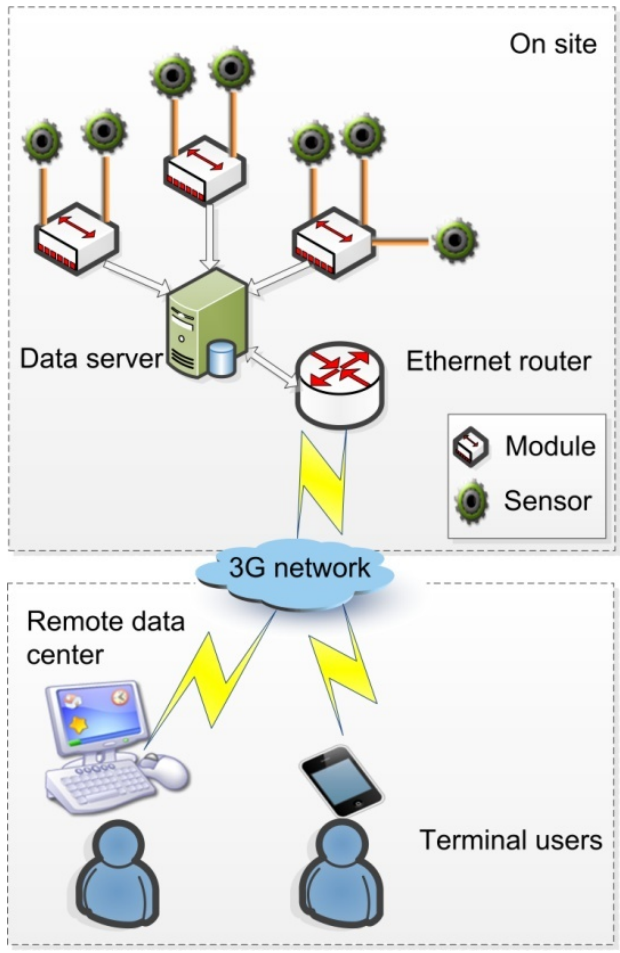

FIGURE 1. Framework of a web-based data acquisition system.

\section{Program Demonstration of the Data Acquisition System}

The data acquisition program was written in the LabVIEW graphical programming language (National Instruments, Austin, TX, USA). The program was installed on a data server (PowerEdge R710, Dell, Round Rock, TX, USA) with two Xeon quad-core processor E5520 (8 M Cache, 2.26 $\mathrm{GHz}$ ) and $8 \mathrm{~GB}$ RAM (random access memory). The main user interface of the program includes four sections: program management panel, data acquisition setting panel, data preview panel, parameter configuration panel, as shown in Fig. 2. In the program management panel, the users can set the acquisition interval and manually stop the program. In the data acquisition setting panel, the users can add more channels for data acquisition, select data collection points, and modify the Internet Protocol (IP) address of the modules. The data preview panel is used to display real-time environmental parameters, acquisition information of date-time, house-identification, and collection-position. In addition to the above panels, most importantly, the program can use the parameter configuration panel to input the measurement range and output type (4-20 mA, 0-5 V, or any other types) of the various sensors. Measured values $x_{i}$ were calculated by:

$$
x_{i}=\frac{\left(S_{i}-4\right) \times\left(R_{H}-R_{L}\right)}{16}+R_{L}
$$

Where,

$x_{i}$ is the ith measurement value, unit is defined by the measured parameter;

$S_{i}$ is the ith analog output value received from the module, units in $\mathrm{mA}$;

$R_{H}$ is the sensor measurement range at the high end, unit defined by the measured parameters, and

$R_{L}$ is the sensor measurement range at the low end, same unit as $R_{H}$. Both $R_{H}$ and $R_{L}$ are user-configurable for each sensor.

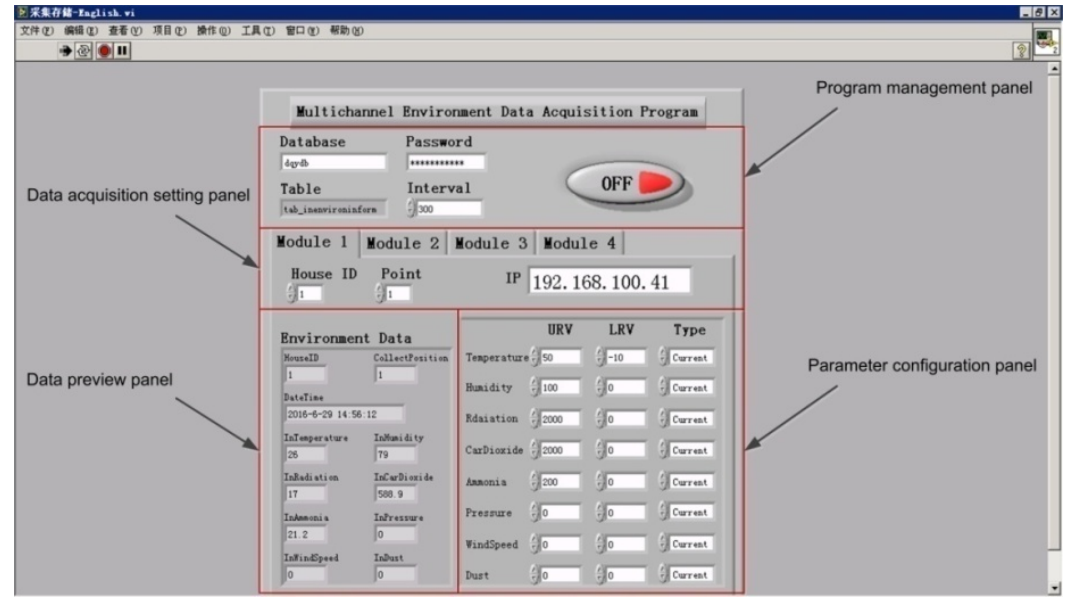

FIGURE 2. Main graphical user interface of the program.

\section{Data Evaluation System with Standard Measuring Instruments}

For evaluation of the measured data, a measurement system with standard instruments (short for System 2) was designed to collect the environmental parameters. The air temperature and relative humidity were recorded by Thermo-Recorder $\left[-30-80{ }^{\circ} \mathrm{C}\left(-22{ }^{\circ} \mathrm{F}-176{ }^{\circ} \mathrm{F}\right), 0.1{ }^{\circ} \mathrm{C}(32.2\right.$ $\left.{ }^{\circ} \mathrm{F}\right)$ sensitivity, $\pm 0.3^{\circ} \mathrm{C}\left( \pm 32.5^{\circ} \mathrm{F}\right)$ resolution; $10-95 \%, 0.1 \%$ sensitivity, $\pm 2.5 \%$ resolution] (RS-13H, ESPEC MIC Corp, Osaka, Japan). The $\mathrm{CO}_{2}$ concentration was measured using a portable probe [0-2000 ppm, 1-ppm sensitivity, ( $\pm 30 \mathrm{ppm}$ $\pm 2 \%$ reading)-resolution] (GM 70, Vaisala Oyj, Vantaa, Finland). The data was downloaded from the instruments as reference material. Before the experiment, all the sensors and instruments were calibrated to ensure accuracy and reliability of the measured data. 


\section{MATERIAL AND METHODS}

\section{Animals and Housing}

In this study, the experiment was conducted at the Shangzhuang Experimental Station (Haidian District, Beijing, China). A group of 90 laying hens (Hy-Line ${ }^{\circledR}$ Variety Brown, U.S. standard) obtained from a commercial farm (De Qingyuan Agricultural Farm, Yanqing District, Beijing, China) were used. The birds were 32-weeks old when the experiment started on June 27, 2016 and were housed in a pen with the perch system, shown in Figs. 3(a) and (b). The perch system is located in an individual compartment, measuring 4.5 $\mathrm{m} \times 1.5 \mathrm{~m} \times 3.0 \mathrm{~m}(1 \times \mathrm{W} \times \mathrm{h})$ and placed on an elevated plastic grid. It has two lines of drinking water, two stages of food platform, two laying nests, and six perches. The system has two feed lines and two nipple drinker lines, ensuring an even distribution of birds and sufficient activities within the system. Easy access to the nest box through the step perch and soft nest with the grass mat ensures high acceptance among the hens. The system has six perches with an average distance of 300 $\mathrm{mm}$ and a ground angle of $40^{\circ}$, and the birds can easily move between the perch levels owing to the step design. The manure can be cleaned by manure belts beneath the plastic grid to collect the droppings for healthy hens. During this experiment, the light period fell between 05:00 $\mathrm{h}$ and 21:00 h. Feed and water were available ad libitum. The normal management practice was performed, with one visit (less than $300 \mathrm{~s}$ ) in the morning and another one (less than $300 \mathrm{~s}$ ) in the afternoon. During the visits, the caretaker inspected the flock and pen and examined the feed and water.

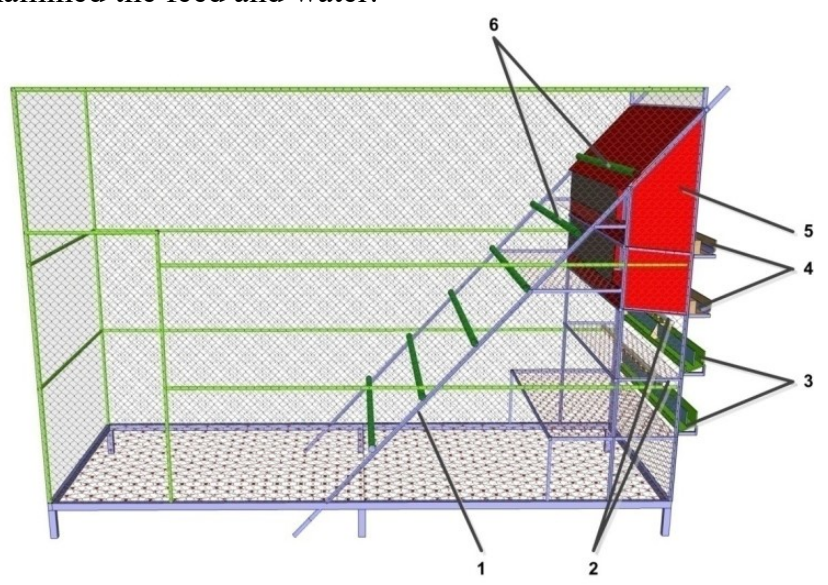

1-perch system, 2-water line, 3-feed trough, 4-egg collection, 5-nest box, 6-perch

(a) System design

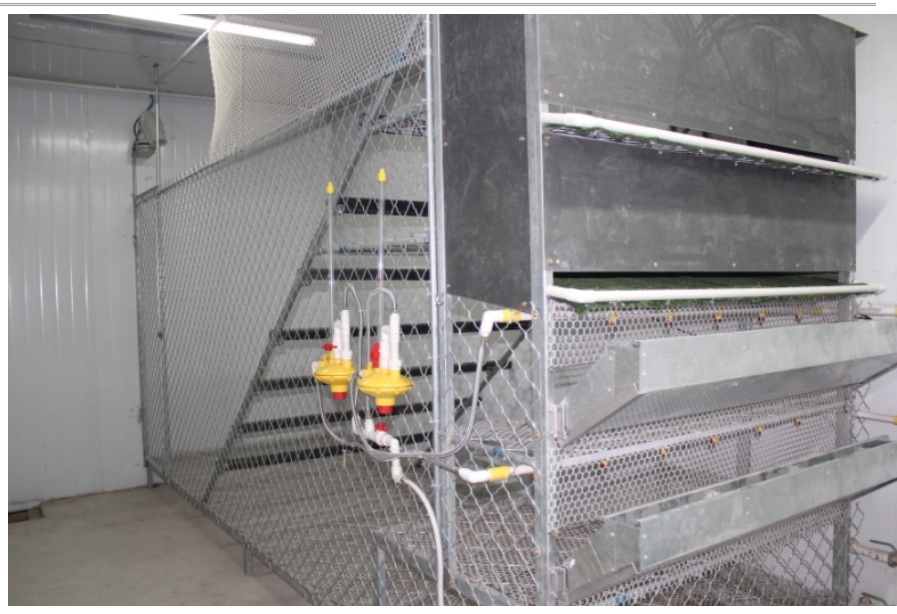

(b) System figure

FIGURE 3. Photos of the perch system for laying hens.

\section{Data Collection}

Two datasets were collected. One was with the sensor data measured by the web-based data acquisition system (System 1) as the calculated values for the uncertainty analysis. The other one was with the reference data recorded by the measurement system (System 2) with standard instruments after calibration as the true values for the data evaluation. Both data were collected simultaneously in the same pen with the same birds under the same configuration. The sources of data collection were the main environmental parameters of the laying hen houses including the air temperature, relative humidity, and carbon dioxide concentration. For the evaluation of the measurement errors, the same data were recorded continuously for 7 days with an acquisition interval of $5 \mathrm{~min}$ when the laying hens were between 32 and 33-weeks old.

\section{Methods of Uncertainty Analysis for Sensors Data}

The usefulness of this data acquisition system for poultry production depends on the performances of the sensors. Therefore, the calibration of the sensors and uncertainty analysis of the sensor data must be conducted completely to maximize the reliability of this system. The traditional procedures of the statistical approaches are strongly dependent on specific assumptions. A complete uncertainty budget has to be calculated for each sensor and any other effects of the instability sources fulfilling the standards of GUM. The measured values in this system depend on the calibration task of the instruments, signal sampling process of the module, data processing with the data server, and any other influences for each sensor. The relevant uncertainty sources are shown in the cause and effect diagram in Fig. 4. 


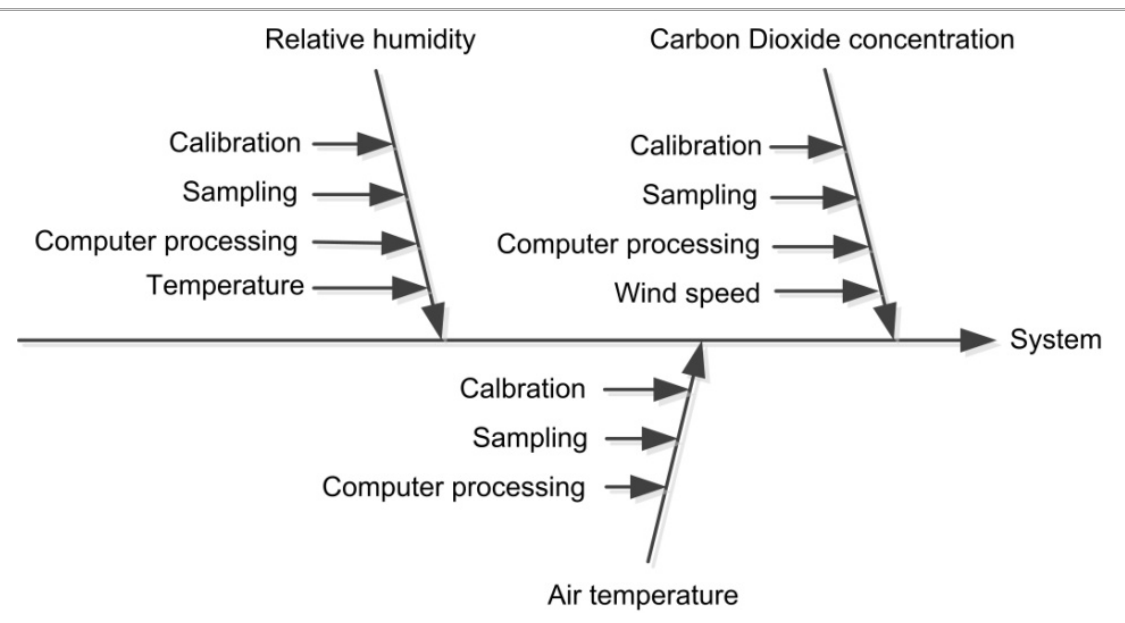

FIGURE 4. Cause and effect diagram with the uncertainty sources of the data acquisition system.

\section{Calibration Procedure and Calculation Algorithm}

A solution is to digitalize the uncertainty sources using mathematical modeling. The general model for the evaluation of the system performances depending on input $n$ quantities of each sensor can be given in the following form:

$$
\begin{aligned}
& u(y)=F\left(X_{1}, X_{2}, \mathrm{~L}, X_{n}\right) \\
& \Delta x_{i}=X_{i}-X_{s}
\end{aligned}
$$

Where,

$X_{i}$ are the measured values obtained from each sensor used in the web-based data acquisition system over the measurement period, and

$X_{s}$ are the true values derived from the measurement system with standard instruments after calibration.

Typically, the arithmetic mean, standard deviation, and measurement error are used to combine the results of the test. The Bessel formula to estimate the sample is

$$
\begin{aligned}
& s_{x_{i}}=\sqrt{\frac{1}{n-1} \sum_{i=1}^{n}\left(\Delta x_{i}-\overline{\Delta x}_{i}\right)^{2}} \\
& \overline{\Delta x}_{i}=\frac{1}{n} \sum_{k=1}^{n} \Delta x_{i}
\end{aligned}
$$

Where,

$\Delta x_{i}$ is the measurement error;

$\overline{\Delta x}_{i}$ is the arithmetic mean of the measurement error;

$n$ is the sampling quantity,

$S_{x_{i}}$ is the standard deviation of the measurement error.

Following the standards of GUM, term $s_{x_{i}}$ represents the uncertainty of the method of type A evaluation. Value $u_{A}$ was determined by the measurement error between System 1 and 2, which can be calculated by [eq. (4)].

In this uncertainty analysis, the method of type B evaluation is related to the accuracy of the sensors, precision of the measuring instruments, data processing approaches, and any other effects, as shown in Fig. 4. The value $\left(u_{B}\right)$ of the evaluation method can be expressed as

$$
u_{B}=\sqrt{u_{B 1}^{2}+u_{B 2}^{2}+\mathrm{L}+u_{B n}^{2}}
$$

Expanded uncertainty refers to the $95 \%$ coverage interval of the measurement results, which is a key component in the uncertainty evaluation of the effective calibration. This function can be written as

$$
u=k \sqrt{u_{A}^{2}+u_{B}^{2}}
$$

Where,

$\mathrm{k}$ is the coverage factor (usually $\mathrm{k}=2$ ), and

$\mathrm{u}_{\mathrm{A}}$ and $\mathrm{u}_{\mathrm{B}}$ are the standard uncertainties of the measured values for each sensor in this system.

For a multiplicative expression, the relative uncertainties of this data acquisition system are as follows:

$$
U=\sqrt{\left(\frac{u_{X_{1}}}{X_{1}}\right)^{2}+\left(\frac{u_{X_{2}}}{X_{2}}\right)^{2}+\mathrm{L}+\left(\frac{u_{X_{n}}}{X_{n}}\right)^{2}}
$$

\section{RESULTS AND DISCUSSION}

\section{Uncertainty Analysis of the Sensors Data}

The following example will illustrate the procedures of the uncertainty analysis suggested by the standards of GUM. Fulfilling the requirements of healthy breeding and animal welfare for laying hens, three main environmental parameters were considered in the system evaluation and uncertainty analysis. In addition to the relevant uncertainty sources described in Fig. 4, all the other influences are assumed to be negligible to keep the calculation concise. The results of the uncertainty analysis following the calculation scheme for each sensor are listed in Table 2. The evaluation is performed with the mean measurement error for each sensor, and the reference data are recognized as more accurate than the data collected by System 1 presented in Table 1 . In this data acquisition system, the stability of the air temperature is calculated as $0.49{ }^{\circ} \mathrm{C}$, mean measurement error of the relative humidity is limited to $0.72 \%$ by Equation (4), and measurement uncertainty of $\mathrm{CO}_{2}$ concentration for the methods of type $\mathrm{A}$ and $\mathrm{B}$ evaluation multiplied by the coverage factor $(\mathrm{k}=2)$ is $67.8 \mathrm{ppm}$. 
TABLE 2. Uncertainty analysis of the various sensor data following the standards of GUM.

\begin{tabular}{lccccccc}
\hline & $\begin{array}{c}\text { Sample } \\
\text { size (n) }\end{array}$ & $\begin{array}{c}\text { Measurement } \\
\text { error }\left(\overline{\Delta \boldsymbol{x}_{i}}\right)\end{array}$ & $\begin{array}{c}\text { Type A } \\
\left(\mathrm{u}_{\mathrm{A}}\right)\end{array}$ & $\begin{array}{c}\text { Type B } \\
\left(\mathrm{u}_{\mathrm{B}}\right)\end{array}$ & $\begin{array}{c}\text { Coverage } \\
\text { factor }(\mathrm{k})\end{array}$ & $\begin{array}{c}\text { Expanded } \\
\text { uncertainty }(\mathrm{u})\end{array}$ & $\begin{array}{c}\text { Relative uncertainty } \\
\left(\mathrm{u}_{\mathrm{r}}\right)\end{array}$ \\
\hline Air temperature $\left({ }^{\circ} \mathrm{C}\right)$ & 1440 & 0.49 & 0.48 & 0.18 & 2 & 1.02 & $4.3 \%$ \\
Relative humidity $(\%)$ & 1440 & 0.72 & 2.29 & 1.55 & 2 & 5.54 & $11.4 \%$ \\
$\mathrm{CO}_{2}$ concentration $(\mathrm{ppm})$ & 1440 & 49.4 & 22.6 & 25.3 & 2 & 67.8 & $10.2 \%$ \\
\hline
\end{tabular}

\section{Estimation of the Data Acquisition System}

From the cause and effect with uncertainty sources of this system (Figure 4), it is concluded that the sensitivity and uncertainty of environmental sensors could be used to examine the system performance and stability. Adding the relative uncertainties of the three environmental parameters, the total uncertainty of this system in accordance with the standards of GUM is given in [eq. (9)]:

$U_{\text {total }}=\sqrt{\left(\frac{u_{X_{1}}}{X_{1}}\right)^{2}+\left(\frac{u_{X_{2}}}{X_{2}}\right)^{2}+\left(\frac{u_{X 3}}{X_{3}}\right)^{2}}=\sqrt{4.3^{2}+11.4^{2}+10.2^{2}}=15.9 \%$

As listed in Table 2, the measurement uncertainties of this data acquisition system mainly originate from the sensors of relative humidity and $\mathrm{CO}_{2}$ concentration having a higher relative uncertainty $\left(\mathrm{u}_{\mathrm{r}}\right)$. Relative humidity sensors employing a moisture sensitive device (MSD) are commonly used for monitoring a wide range of application sectors. However, the long-term stability of this sensing device is still a major challenge owing to environmental pollution and static electricity (Chen \& Lu, 2005; Tripathy et al., 2014). In addition, based on the multi-point air sampling and analyzing system (Ni et al., 2010) and wide applications of electrochemical-based sensors for $\mathrm{CO}_{2}$ sensing methods (Behera et al., 2015), the $\mathrm{CO}_{2}$ concentration measurement can be easily disturbed by airflow fluctuation and human operation. Hence, very precise measurements and error evaluation were needed and adopted to elicit the disturbances and provide excellent stability.

During the process of data evaluation and uncertainty analysis, Monte Carlo methods have shown to be a reasonable tool for uncertainty analysis and delivering realistic results when the model is highly complex (Kraan, 2015). From the comparisons, it is obvious that the methods of type A and B evaluation following the standards of GUM are appropriate for the evaluation using the data acquisition system. The corresponding probability distribution functions (PDFs) could not be retrieved between the data acquisition system and various sensors. Such a model is invalid for uncertainty analysis and sensor network evaluation because the relationships between the data acquisition system and sensors are unknown (Behrens, 2010; Ljungblad et al, 2011; Renault \& Scheeres, 2015). Thus, the algorithm following GUM for the uncertainty analysis proves to be efficient for evaluating the performances of the sensor networks and data acquisition system. From the case study in this research, it is concluded that calibration of the sensors and reduplicated measurements are essential and indispensable to eliminate the systematic errors and standard deviation during the data acquisition process. Every effort must be taken to ensure that this system is appropriate for environment monitoring and poultry management by decision makers while simultaneously considering the multiple sources of uncertainty (Calvet et al., 2013; Leinonen et al., 2016).

\section{CONCLUSIONS}

In this research, a web-based data acquisition system using the LabVIEW software program for poultry management was developed and an uncertainty analysis of various sensor data was proposed for error correction. The results indicated that most of the instabilities sources were related to the measurement activities of the relative humidity and $\mathrm{CO}_{2}$ concentration. The total uncertainty of this system was estimated as $15.9 \%$, fulfilling the standards of GUM for current performances. To improve the system accuracy, the standards of GUM were used to provide the calculated mobility with the measured data. The precision control of this system was achieved by obtaining accurate data from the sensors. To improve animal management, high-level algorithms of uncertainty analysis are being tested at the supervisory level. Through repeated calibration and practical application in commercial farms, this system could be an excellent management tool for poultry production, which will increase the profitability and quality.

\section{ACKNOWLEDGMENTS}

The authors wish to appreciate the support from the National Natural Science Foundation of China (Grant No. 31402113). We are grateful to Prof. Yuan-Hui Zhang from the University of Illinois at Urbana-Champaign (UIUC) for the careful revisions and to Prof. Bao-Ming Li from China Agricultural University for his helpful suggestions. The authors also thank the Beijing De Qingyuan Agricultural Farm and Shangzhuang Experimental Station for their cooperation and assistance in this study.

\section{REFERENCES}

Behera K, Pandey S, Kadyan A, Pandey S (2015) Ionic liquid-based optical and electrochemical Carbon Dioxide sensors. Sensors 15(12):30487-30503.

Behrens R (2010) Uncertainties in external dosimetry: analytical vs. Monte Carlo method. Radiation Protection Dosimetry 138(4):346-352.

BIPM - International Bureau of Weights and Measures (2015) Joint Committee for guides in metrology (JCGM) guide: Evaluation of measurement, Official version. Available in: www.bipm.org. 
Broekhuizen H, Groothuis-Oudshoorn CGM, van Til JA, Hummel JM, Ijzerman MJ (2015) A review and classification of approaches for dealing with uncertainty in multi-mriteria decision analysis for healthcare decisions. Pharmaco Economics 33(5):445-455.

Brunner P, Doherty J, Simmons CT (2012) Uncertainty assessment and implications for data acquisition in support of integrated hydrologic models. Water Resource Research 48(7):W07513.

Calvet S, Gates RS, Zhang GQ, Estelles F, Ogink NWM, Pedersen S, Berckmans D (2013) Measuring gas emissions from livestock buildings: A review on uncertainty analysis and error sources. Biosystems Engineering 116(3):221-231.

Chen CY, Wang C, Wen HQ, Wang L, Jin Y (2016)

Development of adaptive Monte Carlo method software for the evaluation of uncertainty measurement. Journal of China Jiliang University 27(4):406-410. DOI:

http://dx.doi.org/10.3969/j.issn. 2096-2835.2016.04.009 (in Chinese)

Chen Z, Lu C (2005) Humidity sensors: A review of materials and mechanisms. Sensor Letters 3(4):274-295.

Datla RV, Kessel R, Smith AW, Kacker RN, Pollock DB (2010) Uncertainty analysis of remote sensing optical sensor data: Guiding principles to achieve metrological consistency. International Journal Remote Sensing 31(4):867-880.

Ellison SLR, Williams A (2012) Eurachem/CITAC guide: quantifying uncertainty in analytical measurement. $3^{\text {rd }} \mathrm{ed}$. ISBN 978-0-948926-30-3. Available in: www.eurachem.org.

GAQS - General Administration of Quality Supervision (1999) Measurement technical specification: Evaluation and expression of uncertainty in measurement, JJF1059-1999. Available in: www.aqsiq.gov.cn.

Georgiadis P, Cavouras D, Sidiropoulos K, Ninos K, Nomicos C (2009) Remote monitoring of electromagnetic signals and seismic events using smart mobile devices. Computers Geosciences 35(6):1296-1303.

Huang SL, Ge LY, Wang ZY, Zhao W (2011) Poor information material Brinell hardness measurement uncertainty evaluation based on Monte Carlo method. Transactions of the Chinese Society of Agricultural Machinery 42(9):225-229. DOI: http://dx.doi.org/10.3969/j.issn.1000-1298.2011.09.043 (in Chinese)

ISO - International Organization for Standardization (1995) Guide to the expression of uncertainty in measurement. $1^{\text {st }}$ ed. Available in: www.iso.org.

JCGM - Joint Committee for Guides in Metrology (2015) JCGM 100 series: Guides to the expression of uncertainty in measurement, ISO/IEC Guide 98. Available in: www.iso.org/sites/JCGM/GUM -introduction.htm.

Kessel R, Berglund M, Wellum R (2008) Application of consistency checking to evaluation of uncertainty in multiple replicate measurements. Accreditation Quality Assurance 13(6):293-298.

Kraan AC (2015) Range verification methods in particle therapy: Underlying physics and Monte Carlo modeling. Frontiers Oncology 5:150.
Leinonen I, Williams AG, Kyriazakis I (2016) Comparing the environmental impacts of UK turkey production systems using analytical error propagation in uncertainty analysis. Journal of Cleaner Prodution 112(1):141-148.

Ljungblad S, Josefson LE, Holmsten M (2011) Method for effective calibration of temperature loggers with automated data sampling and evaluation. International Journal Thermophysics 32(11):2495-2503.

Pechlivanidis IG, Jackson BM, Mcintyre NR, Wheater HS (2011) Catchment scale hydrological modeling: A review of model types, calibration approaches and uncertainty analysis methods in the context of recent developments in technology and applications. GlobalNEST International Journal 13(3):193-214.

Reilly M, Willenbockel D (2010) Managing uncertainty: A review of food system scenario analysis and modelling. Philosophical Transactions of the Royal Society B: Biological Sciences 365(1554):3049-3063.

Renault CA, Scheeres DJ (2015) Statistical analysis of control maneuvers in unstable orbital environments. Journal of Guidance, Controls, and Dynamics 26(5):758-769.

Sarachi S, Hsu KL, Sorooshian S (2015) A statistical model for the uncertainty analysis of satellite precipitation products. Journal Hydrometeorology 16(5):2101-2117.

Serodio C, Cunha JB, Morais R, Couto C, Monteiro J (2001) A networked platform for agricultural management systems. Computers and Electronics Agriculture 31(1):75-90.

Snow MG, Bajaj AK (2015) Uncertainty quantification analysis of the dynamics of an electrostatically actuated microelectromechanical switch model. Journal of Sound and Vibration 349:375-388.

So-In C, Poolsanguan S, Rujirakul K (2014) A hybrid mobile environmental and population density management system for smart poultry farms. Computers and Electronics Agriculture 109:287-301.

Tripathy A, Pramanik S, Cho J, Santhosh J, Osman NAA (2014) Role of morphological structure, doping, and coating of different materials in the sensing characteristics of humidity sensors. Sensors 14(9):16343-16422.

Venkatraman K, Reddy BD, Selvan MP, Moorthi S, Kumaresan N, Gounden NA (2016) Online condition monitoring and power management system for standalone micro-grid using FPGAs. IET Generation, Transmission, Distribution 10(15):3875-3884.

Yustres A, Asensio L, Alonso J, Navarro V (2012) A review of Markov Chain Monte Carlo and information theory tools for inverse problems in subsurface flow. Computational Geosciences 16(1):1-20.

Zahedi ZM, Akbari R, Shokouhifar M, Safaei F, Jalali A (2016) Swarm intelligence based fuzzy routing protocol for clustered wireless sensor networks. Expert System Application 55:313-328.

Zakharov IP, Vodotyka SV, Klimova KA, Shevchenko NS (2013) Some examples of the evaluation of measurement uncertainty. Measurement Technologies 56(6):591-598. 